\title{
In situ hybridization for the detection of the apxIV gene in the lungs of pigs experimentally infected with twelve Actinobacillus pleuropneumoniae serotypes
}

\author{
Wan-Seob CHO, Changsun CHOI, Chanhee CHAE* \\ Department of Veterinary Pathology, College of Veterinary Medicine and School of Agricultural \\ Biotechnology, Seoul National University, Suwon 441-744, Kyounggi Do, Republic of Korea
}

(Received 6 December 2001; accepted 25 March 2002)

\begin{abstract}
The detection of the apxIV gene in lung tissues from pigs experimentally infected with the 12 major A. pleuropneumoniae serotype (1 to 12) reference strains was studied by in situ hybridization using a non-radioactive digoxigenin-labeled DNA probe. In situ hybridization produced a distinct positive signal in all pigs inoculated with the $12 \mathrm{~A}$. pleuropneumoniae serotypes. Positive hybridization typically exhibited a dark-brown to black reaction product in intracellular and extracellular locations, without background staining. A strong hybridization signal was seen in degenerated alveolar leukocytes ("oat cells") adjacent to the foci of coagulative necrosis and in the alveolar spaces. The in situ hybridization methodology developed for the detection of the apxIV gene is a valuable tool for the diagnosis of porcine pleuropneumonia caused by A. pleuropneumoniae when only formalin-fixed tissues are submitted for diagnosis.
\end{abstract}

\section{Actinobacillus pleuropneumoniae / Apx toxin / in situ hybridization / lung / pig}

Résumé - Détection par hybridation in situ du gène apxIV dans les poumons de porcs infectés expérimentalement par douze sérotypes de Actinobacillus pleuropneumoniae. La détection du gène apxIV dans les tissus pulmonaires de porcs infectés expérimentalement par les douze sérotypes majeurs des souches de référence (1 à 12) de Actinobacillus pleuropneumoniae a été effectuée par hybridation in situ utilisant une sonde à $\mathrm{ADN}$ non radioactive marquée à la digoxigénine. L'hybridation in situ a produit un signal positif distinct chez tous les porcs inoculés par les douze sérotypes de $A$. pleuropneumoniae. Un résultat positif d'hybridation entraînait typiquement un produit de réaction marron foncé à noir localisé en intra- et en extra-cellulaire, sans bruit de fond. Un signal fort d'hybridation a été observé dans les leucocytes alvéolaires dégénérés adjacents aux foyers de nécrose et dans les espaces alvéolaires. La méthode d'hybridation in situ développée pour la détection du gène apxIV est un outil précieux pour le diagnostic de la pleuropneumonie porcine causée par A. pleuropneumoniae lorsque seuls les tissus fixés dans le formol sont soumis pour le diagnostic.

Actinobacillus pleuropneumoniae / Apx toxine / hybridation in situ / poumon / porc

*Correspondence and reprints

Tel: (82) 31290 2736; fax: (82) 31294 4588; e-mail: swine@plaza.snu.ac.kr 


\section{INTRODUCTION}

Porcine pleuropneumonia, caused by Actinobacillus pleuropneumoniae, is a highly contagious, fibrinous, hemorrhagic and necrotizing pneumonia that causes high mortality in acutely infected pigs and localized lung lesions in pigs that are chronically infected [24]. A. pleuropneumoniae is detected primarily within neutrophils and alveolar macrophages at the periphery of foci of acute coagulative necrosis and at the edge of granulation tissue in chronic lesions [14]. Based on nicotinamide adenine dinucleotide (NAD) requirements, NADdependent strains (biotype 1) and NAD-independent strains (biotype 2) can be distinguished [20]. To date, at least $15 A$. pleuropneumoniae serotypes (1 to 15 ) have been described, with serotypes 1 and 5 being further subdivided into subtypes $\mathrm{A}$ and B $[2,11,18,19]$. Serotypes 2 and 5 are the most prevalent isolates recovered from acute cases of pleuropneumonia in Korea [15].

Reference strains for the 15 serotypes of A. pleuropneumoniae produce two or three of the Apx-encoded toxins. ApxI is produced by serotypes 1, 5, 9, 10, 11 and 14, and ApxII is produced by all serotypes except serotype 10 and 14 . ApxIII is produced by serotypes $2,3,4,6,8$, and 15 [2, 8, 21], and the ApxIV toxin is produced by all 15 serotypes [2, 21, 22]. The apxIV gene is species-specific rather than serotype-specific $[5,22]$, and because of this, the apxIV gene may serve as a sensitive and specific probe for the diagnosis of $A$. pleuropneumoniae infection.

The objective of the present study was to detect and localize $A$. pleuropneumoniae by means of the apxIV gene in lung tissues from pigs experimentally infected with the 12 major A. pleuropneumoniae serotypes (1 to 12 ) by the in situ hybridization technique using a non-radioactive digoxigeninlabeled DNA probe that targets the apxIV gene of $A$. pleuropneumoniae.

\section{MATERIALS AND METHODS}

\subsection{Bacterial strains}

The A. pleuropneumoniae reference strains used were serotype 1, S4074; serotype 2, S4226; serotype 3, S1421; serotype 4, M62; serotype 5a, K17; serotype 5b, L20; serotype 6, Femo; serotype 7, WF83; serotype 8, 405; serotype 9, CVJ13261; serotype 10, D13039; serotype 11, 56153; and serotype 12, 8329, respectively. All reference serotypes and rabbit antisera against the 12 serotypes were provided by K.R. Mittal, University of Montreal, Saint-Hyacinthe, Quebec, Canada.

\subsection{Experimental design}

Thirty-nine 7-week-old colostrum-deprived pigs housed in an isolation facility were randomly allocated to an infected ( $n=$ $26)$ or non-infected group $(n=13)$. In the infected group, 2 pigs each were inoculated intratracheally with a single serotype of $A$. pleuropneumoniae. A. pleuropneumoniae serotypes were washed once with phosphate-buffered saline (PBS, pH 7.4) and diluted in PBS containing 5\% bovine serum albumin to approximately $10^{6} \mathrm{CFU} / \mathrm{mL}$. The pigs were anesthetized intramuscularly with $2.5 \mathrm{mg}$ of xylazine and $12 \mathrm{mg}$ of ketamine $\mathrm{HCl}$ per $\mathrm{kg}$. Five milliliters of $10^{6} \mathrm{CFU} / \mathrm{mL}\left(5 \times 10^{6} \mathrm{CFU}\right)$ were inoculated into each pig in the infected group. Bacterial plate counts were performed immediately before and after experimental infection to determine the viable-cell counts. Thirteen control pigs were inoculated in the same manner with a sterile medium. All infected and non-infected pigs were euthanized at 18 hours postinoculation.

\subsection{Bacterial isolation}

The lungs from the pigs experimentally infected with the $12 \mathrm{~A}$. pleuropneumoniae serotypes, were cultured for A. Pleuropneumoniae by searing the tissue with a heated 
spatula, incising the tissue with a sterile scalpel, and sampling the exposed area with a sterile cotton swab. The swabs were used to inoculate 2 sheep blood (5\%) agar plates by streaking the plates. A nurse colony of Staphylococcus intermedius was crossstreaked on 1 of the plates. The plates with and without the nurse substreaks were incubated at $37^{\circ} \mathrm{C}$ in a $5 \% \mathrm{CO}_{2}$ incubator for 18-24 h. Identification of the strains as $A$. pleuropneumoniae was based on the Gram-stain reaction, positive hemolysis on 5\% sheep blood agar, positive ChristieAtkins-Munch-Petersen (CAMP) reaction, requirement for NAD, urease production, xylose and mannose fermentation. Negative esculin, trehalose and sorbitol reactions were also considered for NADindependent strains. The CAMP reaction was determined on 5\% sheep blood agar, using a strain of $S$. intermedius producing beta-hemolysis. All isolates were serotyped using the slide agglutination procedure as previously described $[15,17]$.

\subsection{Polymerase chain reaction}

All isolates were examined for the apxIV gene. The PCR for the apxIV gene was carried out as previously described [22], with slight modification. The forward and reverse primers were 5'-TGGCACTGACGGTGATGAT-3' (nucleotides 6018 to 6036) and 5'GGCCATCGACTCAACCAT-3' (nucleotides 6459 to 6442), respectively. Bacterial DNA amplification was carried out in a $50 \mu \mathrm{L}$ reaction mixture containing $5 \mu \mathrm{L}$ of the supernatant of lysed bacteria, $90 \mathrm{ng}$ oligonucleotide primers, $0.2 \mathrm{mM}$ each of dATP, dGTP, dCTP, and dTTP, $10 \mathrm{mM}$ Tris $\mathrm{HCl}$ (pH 8.8), $1.5 \mathrm{mM} \mathrm{MgCl} 2,50 \mathrm{mM} \mathrm{KCl}$, and 1 unit of Taq polymerase, and distilled water to complete to $50 \mu \mathrm{L}$. The PCR profile used included a denaturing step at $94{ }^{\circ} \mathrm{C}$ for $30 \mathrm{~s}$, followed by annealing of the primers at $52{ }^{\circ} \mathrm{C}$ for $30 \mathrm{~s}$, with an extension step at $72{ }^{\circ} \mathrm{C}$ for $30 \mathrm{~s}$. The thirty-five cycles of this 3-step procedure were performed in a thermal cycler, followed by a $10 \mathrm{~min}$ extension at $72{ }^{\circ} \mathrm{C}$.

The amplified product was visualized by standard gel electrophoresis with $10 \mu \mathrm{L}$ of the final reaction mixture on a $2 \%$ agarose gel. Amplified DNA fragments of specific sizes were located by ultraviolet fluorescence after staining with ethidium bromide. Their lengths were verified with a digested lambda DNA standard run simultaneously. The PCR reactions were repeated three times. Control DNA from the reference strain was included in each reaction.

\subsection{Preparation of the labeled probe}

A 442 base pair fragment of the apxIV gene was used as a probe and for its detection, PCR was carried out as previously described [22]. PCR products were purified using a $30-\mathrm{kDa}$ cutoff membrane ultrafiltration filter. The nucleotide sequences of the purified PCR products were determined using BigDye chemistry with the ABI Prism Sequencer (Applied Biosystems, Foster City, CA, USA). Sequencing was performed on the purified PCR products before PCR products were labeled by random priming with digoxigenindUTP (Boehringer Mannheim, Indianapolis, IN, USA) according to the manufacturer's instructions.

\subsection{In situ hybridization}

Tissues from each pig were collected and placed in $10 \%$ neutral buffered formalin, and after one or two days of fixation, were dehydrated through graded alcohols and a xylene step and were embedded in paraffin wax. Three serial sections $(4 \mu \mathrm{m})$ were prepared from each of the formalinfixed, paraffin wax-embedded tissues. Two were processed for the apxIV gene in situ hybridization without DNase I treatment and one was processed for the apxIV gene in situ hybridization with DNase I treatment. 
Sections for the in situ hybridization were placed on Superfrost/Plus slides (Fisher Scientific, Pittsburgh, PA, USA), and were stored at room temperature. Just before use, they were dewaxed in xylene, rehydrated in phosphate-buffered saline (PBS; pH 7.4, 0.01M) for $5 \mathrm{~min}$, and deproteinized with $0.2 \mathrm{~N} \mathrm{HCl}$ for 20 min at room temperature. They were then digested at $37^{\circ} \mathrm{C}$ for $20 \mathrm{~min}$ in PBS containing $200 \mu \mathrm{g} / \mathrm{mL}$ of proteinase K (Gibco BRL, Grand Island, NY, USA). For each tissue examined, a serial section was treated with DNase I (Boehringer Mannheim) at 0.1 unit $/ \mathrm{mL}$ in $10 \mathrm{mM}$ Tris- $\mathrm{HCl}(\mathrm{pH} 7.4)$ for $30 \mathrm{~min}$ at $37{ }^{\circ} \mathrm{C}$ to remove target DNA, as a specificity control. After digestion, the tissues were fixed in paraformaldehyde $4 \%$ in PBS for $10 \mathrm{~min}$. After rinsing with PBS twice, the slides were acetylated in $300 \mathrm{~mL}$ of $0.1 \mathrm{mM}$ triethanolamine- $\mathrm{HCl}$ buffer (pH 8.0) to which $0.75 \mathrm{~mL}$ of acetic anhydride $(0.25 \%)$ had been added. After $5 \mathrm{~min}$, a further $0.75 \mathrm{~mL}$ of acetic anhydride was added, and $5 \mathrm{~min}$ later the slides were rinsed in $2 \times$ saline sodium citrate $(\mathrm{SSC}) .(1 \times \mathrm{SSC}$ contains $50 \mathrm{mM} \mathrm{NaCl}$ and $15 \mathrm{mM}$ sodium citrate $\mathrm{pH}$ 7.0.)

Hybridization was carried out overnight at $45^{\circ} \mathrm{C}$. The digoxigenin-labeled probe $(1 \mathrm{ng} / \mu \mathrm{L})$ was diluted in $50 \mu \mathrm{L}$ of standard hybridization buffer consisting of $2 \times \mathrm{SSC}$ containing $50 \%$ deionized formamide, $10 \mathrm{mg}$ salmon sperm DNA (Oncor, Gaithersburg, MD, USA), $0.02 \%$ sodium dodecyl sulphate (SDS), 1\% Denhart solution and $50 \%$ dextran sulphate solution (50\% concentration). Approximately, $75 \mathrm{ng}$ of the digoxigenin-labeled probe were added to the standard hybridization buffer (70 $\mu \mathrm{L})$, which was then layered over the section. Fluid was held in place by a coverslip (the edges of which were sealed with rubber cement) and was heated for 10 min on a $95^{\circ} \mathrm{C}$ heating block. After overnight hybridization, the sections were thoroughly washed twice in $4 \times$ SSC for $10 \mathrm{~min}$ at room temperature, twice in $2 \times$
SSC for 10 min at $45^{\circ} \mathrm{C}$, twice in $2 \times \mathrm{SSC}$ for $10 \mathrm{~min}$ at room temperature, twice in $0.2 \times \mathrm{SSC}$ for $10 \mathrm{~min}$, once in maleic acid buffer $(100 \mathrm{mM}$ maleic acid and $150 \mathrm{mM}$ $\mathrm{NaCl}, \mathrm{pH} 7.5$ ) for $5 \mathrm{~min}$ and once in a $1 \times$ blocking reagent (Boehringer Mannheim) for $40 \mathrm{~min}$ at room temperature.

For the detection of hybridization, the sections were incubated with antidigoxigenin conjugated with alkaline phosphatase (Boehringer Mannheim) diluted 1 in 500 in the $1 \times$ blocking reagent. After three washes in the buffer, the substrate consisting of nitroblue tetrazolium (NBT) and 5-bromocresyl-3-indolylphosphate (BCIP) was layered over the sections. Color was allowed to develop for 3-4 h in the dark, and development was stopped by dipping the slides briefly in triethylenediaminetetraacetic acid (EDTA) buffer (10 mM Tris- $\mathrm{HCl}$ and $1 \mathrm{mM}$ EDTA, pH 8.0). The sections were counterstained with methyl green $0.5 \%$, and the slides were then washed with distilled water for $1 \mathrm{~min}$ and dried completely.

Three controls were employed with each in situ hybridization procedure: (i) predigestion of a consecutive tissue section from infected and non-infected pigs with a solution of DNase I before in situ hybridization, (ii) in situ hybridization performed on serial sections of infected tissue with an apxIV DNA probe and (iii) in situ hybridization performed with an apxIV DNA probe on tissues of non-infected pigs.

\section{RESULTS}

\subsection{Bacterial isolation}

The respective $A$. pleuropneumoniae serotypes were isolated from each pig inoculated with $A$. pleuropneumoniae. All isolates were the same serotype as in the previously inoculated strains. A. pleuropneumoniae was not isolated from non-infected pigs. The 12 A. pleuropneumoniae serotypes produced the expected 442 base pair fragment of 
the apxIV gene. Polymerase chain reaction analysis of the $12 \mathrm{~A}$. pleuropneumoniae serotypes isolated from the lungs of experimentally infected pigs demonstrated that every serotype carries the apxIV gene. The results of each of the three PCR reactions were consistent. The PCR products obtained from the respective serotypes were sequenced and their identities were confirmed as the apxIV gene.

\subsection{In situ hybridization}

The sensitivity and specificity of in situ hybridization were determined. The sensitivity of in situ hybridization was determined as the number of in situ hybridization positive lungs divided by the number of $A$. pleuropneumoniae-inoculated pigs; $26 / 26=100 \%$. The specificity was determined by dividing the number of control samples that were negative for in situ hybridization by the number of noninfected pigs: $13 / 13=100 \%$.

In situ hybridization produced a distinct positive signal of the apxIV gene in lung specimens from all pigs inoculated with the 12 A. pleuropneumoniae serotype reference strains. The morphology of the lung tissues was well-preserved (data not shown), despite the relatively high temperature required for the incubation procedure. The signal intensity varied within the tissue sections and between pigs. Positive hybridization typically exhibited a dark-brown to black reaction product at intracellular and extracellular locations and there was no background staining.

A strong hybridization signal was seen in the degenerated alveolar leukocytes ("oat cells") adjacent to the foci of coagulative necrosis and in the alveolar spaces (Figs. 1A and 1B). Less intense signals were seen in the granulation tissues surrounding the foci of necrosis. Neutrophils and macrophages that had infiltrated into the alveolar spaces frequently showed strong hybridization signals. Identification of the cell types containing apxIV genes was occasionally difficult, but examination of the adjacent sections stained with hematoxylin and eosin confirmed that the apxIV genes were present in neutrophils and macrophages. Positive hybridization signals were also detected free within the alveolar spaces. Sections from the non-infected pigs showed no hybridization signals for the apxIV gene.

\section{DISCUSSION}

The results of this study demonstrated that a non-radioactive digoxigenin-labeled DNA probe for the apxIV gene can detect the 12 A. pleuropneumoniae serotype reference strains in formalin-fixed paraffin-embedded tissues. Hitherto, an accurate diagnosis of porcine pleuropneumonia has depended on the isolation of the etiological agent and examination of its biochemical and morphological properties, and on the serological status of the herd [7]. Culture of A. pleuropneumoniae can be relatively insensitive, especially in chronic cases, and it is also time-consuming [16]. In addition, fresh or frozen tissue is not always available for examination. Formalin fixation is the standard method for tissue preservation in veterinary pathology and fixed specimens are the major source of tissues for many studies.

In contrast to ApxI, ApxII and ApxIII cytotoxins, each of which is produced by some but not all serotypes of $A$. pleuropneumoniae $[1,3,6,12]$, the cytotoxin synthesized by the apxIV gene is produced by all serotypes [5, 22]. Furthermore, ApxI and-II are not species specific because they are present in other species of the Pasteurellaceae family [23]. In addition, some field isolates of $A$. pleuropneumoniae do not produce ApxI, ApxII and ApxIII toxins [1, 13, 15], whereas all field isolates, studied so far, carry the apxIV gene $[4,5,21]$. A new study using a PCR-based assay also confirmed the presence of the $A$. pleuropneumoniae 

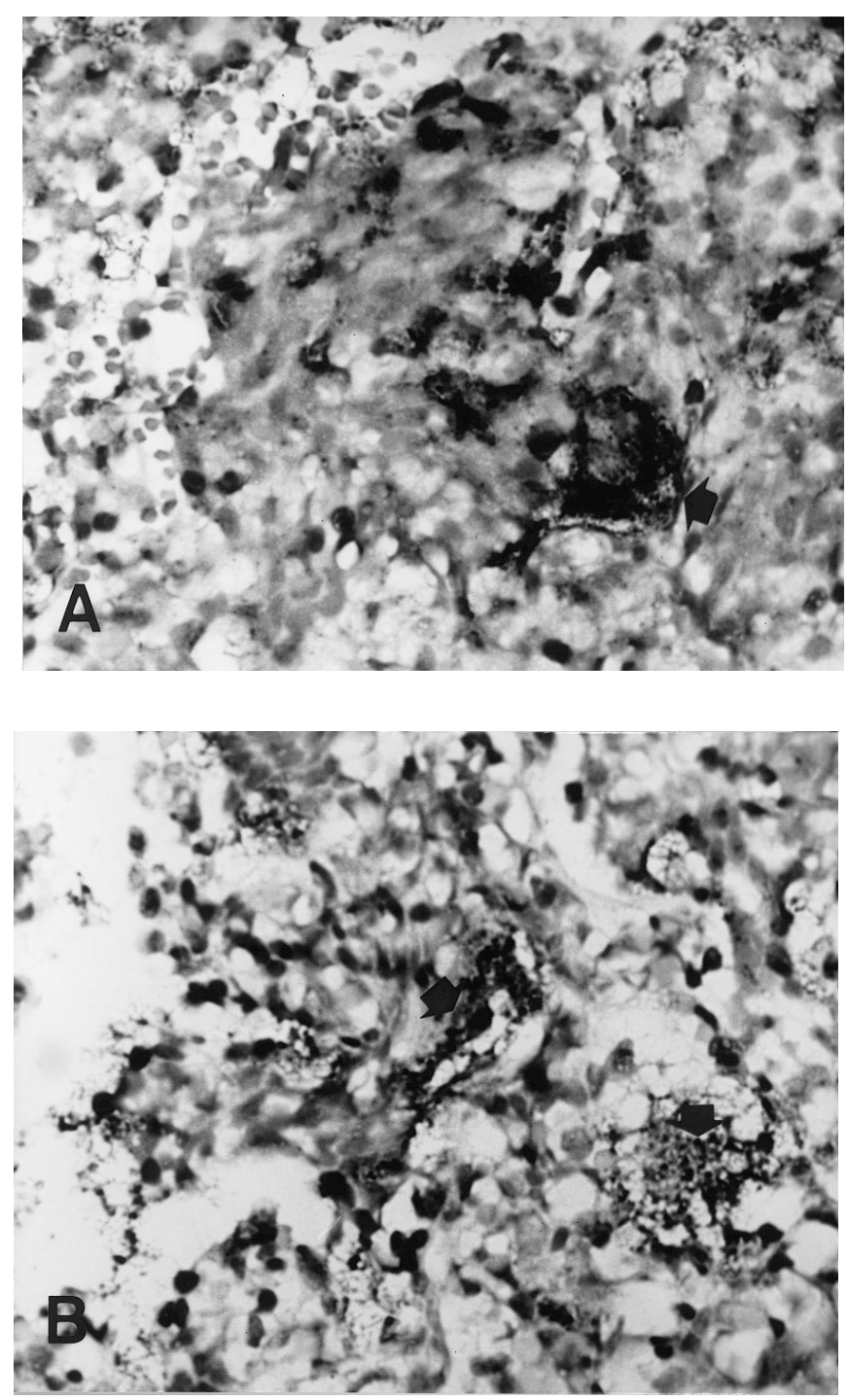

Figure 1. The ap $x I V$ gene (arrows) is demonstrated in streaming degenerated alveolar leukocytes in alveolar spaces from pig experimentally infected with Actinobacillus pleuropneumoniae serotype 2 (A) and A. pleuropneumoniae serotype 5 (B). In situ hybridization, nitroblue tetrazolium/5bromocresyl-3-indolylphosphate, methyl green counterstain $(\times 400)$.

apxIV gene in homogenates from necrotic lesions [21]. Therefore, the apxIV gene is a good choice as a sensitive and specific probe for the diagnosis of $A$. pleuropneumoniae infection.
In situ hybridization preserves cellular and histological detail such that positive hybridization signals and lesions may be examined on the same tissue section. The results of an earlier study indicate that 
fluorescent in situ hybridization using a DNA probe designed from the $16 \mathrm{~S}$ ribosomal RNA of $A$. pleuropneumoniae can detect the 12 serotypes of $A$. pleuropneumoniae $[9,10]$. However, that study did not examine lung tissue from pigs infected with all of the A. pleuropneumoniae serotypes, and the use of fluorescent in situ hybridization did not preserve cellular and histological details. In addition, the use of a fluorescence microscope was necessary, and the hybridized slides could not serve as permanent records because the fluorescence fades quickly.

Considering its technical complexity and expense, in situ hybridization may not be suitable for routine use in diagnostic laboratories at the present time. However, the ease of preparation of DNA probes by PCR combined with the increasing availability of probes and sequence databases should facilitate the use of this technique in diagnostic veterinary medicine and research. The use of a digoxigenin-labeled probe facilitates the transfer of this methodology to diagnostic laboratories that already perform immunohistochemical procedures. In situ hybridization using a digoxigenin labeled probe as described in the present study will allow for the diagnosis of $A$. pleuropneumoniae infection while preserving the morphology of the tissue.

\section{ACKNOWLEDGEMENTS}

The research reported here was supported by the Ministry of Agriculture, Forestry and Fisheries-Special Grants Research Program (MAFFSGRP) and the Brain Korea 21 Project, Republic of Korea.

\section{REFERENCES}

[1] Beck M., Van Den Bosch J.F., Jongenelen I.M.C.A., Loeffen P.L.W., Nielsen R., Nicolet J., Frey J., RXT toxin genotypes and phenotypes in Actinobacillus pleuropneumoniae field strains, J. Clin. Microbiol. 32 (1994) 2749-2754.
[2] Blackall P.J., Klaasen H.L.B.M., Van den Bosch H., Kuhnert P., Frey J., Proposal of a new serovar of Actinobacillus pleuropneumoniae: serovar 15, Vet. Microbiol. 84 (2002) 47-52.

[3] Chang Y.F., Young R., Struck D.K., Cloning and characterization of a hemolysin gene from Actinobacillus (Haemophilus) pleuropneumoniae, DNA 8 (1989) 635-647.

[4] Cho W.-S., Chae C., Expression of the apxIV gene in pigs naturally infected with Actinobacillus pleuropneumoniae, J. Comp. Pathol. 125 (2001) 34-40.

[5] Cho W.-S., Chae C., Genotypic prevalence of apxIV in Actinobacillus pleuropneumoniae field isolates, J. Vet. Diagn. Invest. 13 (2001) 175-177.

[6] Choi C., Kwon D., Min K., Chae C., Detection of localization of ApxI, -II, and -III genes of Actinobacillus pleuropneumoniae in natural porcine pleuropneumonia by in situ hybridization, Vet. Pathol. 38 (2001) 390-395.

[7] Dubreuil J.D., Jacques M., Mittal K.R., Gottschalk M., Actinobacillus pleuropneumoniae surface polysaccharides: their role in diagnosis and immunogenicity, Anim. Health Res. Rev. 1 (2000) 73-93.

[8] Frey J., Beck M., Van Den Bosch J.F., Segers R.P., Nicolet J., Development of an efficient PCR method for toxin typing of Actinobacillus pleuropneumoiae strains, Mol. Cell. Probes 9 (1995) 277-282.

[9] Fussing V., Paster B.J., Dewhirst F.E., Poulsen L.K., Differentiation of Actinobacillus pleuropneumoniae strains by sequence analysis of $16 \mathrm{~S}$ rDNA and ribosomal intergenic regions, and development of a species specific oligonucleotide for in situ detection, Syst. Appl. Microbiol. 21 (1998) 408-418.

[10] Jensen T.K., Boye M., Hagedorn-Olsen T., Riising H.J., Angen O., Actinobacillus pleuropneumoniae osteomyelitis in pigs demonstrated by fluorescent in situ hybridization, Vet. Pathol. 36 (1999) 258-261.

[11] Jolie R.A., Mulks M.H., Thacker B.J., Antigenic differences within Actinobacillus pleuropneumoniae serotype 1, Vet. Microbiol. 38 (1994) 329-349.

[12] Kamp E.M., Popma J.K., Anakotta J., Smits M.A., Identification of hemolytic and cytotoxic proteins of Actinobacillus pleuropneumoniae by use of monoclonal antibodies, Infect. Immun. 59 (1991) 3079-3085.

[13] Kamp E.M., Vermeulen T.M., Smits M.A., Haagsma J., Production of Apx toxins by field strains of Actinobacillus pleuropneumoniae and Actinobacillus suis, Infect. Immun. 62 (1994) 4063-4065.

[14] Min K., Chae C., Detection and distribution of DNA of Actinobacillus pleuropneumoniae in the lungs of naturally infected pigs by in-situ hybridization, J. Comp. Pathol. 119 (1998) 169-175. 
[15] Min K., Chae C., Serotype and apx genotype profiles of Actinobacillus pleuropneumoniae field isolates in Korea, Vet. Rec. 145 (1999) 251-254.

[16] Mittal K.R., Higgins R., Larivière S., Detection of type-specific antigens in the lungs of Haemophilus pleuropneumoniae-infected pigs by coagglutination test, J. Clin. Microbiol. 18 (1983) 1355-1357.

[17] Mittal K.R., Higgins R., Larivière S., Évaluation of slide agglutination and ring precipitation tests for capsular serotyping of Haemophilus pleuropneumoniae, J. Clin. Microbiol. 15 (1982) 1019-1023.

[18] Nielsen R., Serology of Haemophilus (Actinobacillus) pleuropneumoniae serotype 5 strains: establishment of subtypes a and b, Acta Vet. Scand. 27 (1986) 49-58

[19] Nielsen R., Andresen L.O., Plambeck T., Nielsen J.P., Krarup L.T., Jorsal S.E., Serological characterization of Actinobacillus pleuropneumoniae biotype 2 strains isolated from pigs in two Danish herds, Vet. Microbiol. 54 (1997) 35-46.

[20] Pohl S., Bertschinger H.U., Frederiksen W., Mannheim W., Transfer of Haemophilus pleuropneumoniae and the Pasteurella haemolytica- like organism causing porcine necrotic pleuropneumonia to the genus Actinobacillus (Actinobacillus pleuropneumoniae comb. nov.) on the basis of phenotypic and deoxyribonucleic acid relatedness, Int. J. Syst. Bacteriol. 33 (1983) 510-514.

[21] Schaller A., Djordjevic S.P., Eamens G.J., Forbes W.A., Kuhn R., Kuhnert P., Gottschalk M., Nicolet J., Frey J., Identification and detection of Actinobacillus pleuropneumoniae by PCR based on the gene ApxIVA, Vet. Microbiol. 79 (2001) 47-62.

[22] Schaller A., Kuhn R., Kuhnert P., Nicolet J., Anderson T.J., MacInnes J.I., Segers R.P., Frey J., Characterization of apxIV, a new RTX determinant of Actinobacillus pleuropneumoniae, Microbiology 145 (1999) 2105-2116.

[23] Schaller A., Kuhnert P., de la Puente-Redondo V.A., Nicolet J., Frey J., Apx toxins in Pasteurellaceae species from animals, Vet. Microbiol. 74 (2000) 365-376.

[24] Taylor D.J., Actinobacillus pleuropneumoniae, in: Straw B.E., D’allaire S., Mengeling W.L., Taylor D.J. (Eds.), Diseases of Swine, Iowa State University Press, Ames, Iowa, USA, 1999, pp. 343-354

To access this journal online: www.edpsciences.org 